\title{
Hombro doloroso y tumor de Pancoast
}

\author{
F. del Campo Matías*, J. L. González PeláeZ ${ }^{* *}$, J. Mã . Martín Santos*** \\ *Adjunto del Servicio de Neumología. ProfesorAsociado de la Facultad de Medicina \\ de Valladolid. Hospital Universitario Pío del Río Hortega. Valladolid. ${ }^{*}$ Residente de \\ Medicina Familiar y Comunitaria. Unidad de Medicina Familiar y Comunitaria. Hos - \\ pital Universitario Pío del Río Hortega. Valladolid. ${ }^{* * *}$ Adjunto de la Sección de Reu - \\ matología. Profesor Asociado de la Facultad de Medicina de Valladolid. Hospital
}

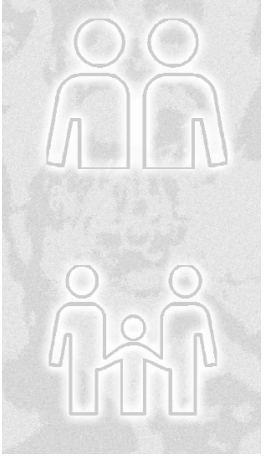

Shoulder pain and Pancoast tumor

\section{RESUMEN}

El tumor de Pancoast es una forma de presentación conocida del carcinoma de pulmón. A pesar de ello, como consecuencia de su forma de manifestación, tanto clínica como radiológica, hace que a menudo no sean diagnosticados correctamente, siendo remitidos inicialmente este tipo de pacientes a la consulta de traumatología, lo que hace que el diagnóstico y tratamiento se retrase innecesariamente.

Palabras clave: Tumor de Pancoast. Hombro

\section{doloroso.}

\section{ABSTRACT}

The Pancoast tumor is a known form of presentation of lung cancer. In spite of it, due to its manifestation way, both clinical and radiographic, makes that this kind of patients often do not be diagnosed correctly, being sent first to the orthopaedics clinics, which produces an unnecessary delay in their diagnosis and treatment.

Key words: Pancoast tumor. Shoulder pain.

\section{INTRODUCCIÓN}

El dolor de hombro constituye una causa habitual de consulta médica en Atención Primaria ${ }^{1}$. Si este dolor se acompaña de semiología de afectación radiculoplexular del brazo, deberemos tener en cuenta la posibilidad de un proceso tumoral a nivel del vértice pulmonar homolateral (tumor de Pancoast). Ello debe obligarnos a un examen minucioso de la radiografía de tórax, dado que si no, podemos infradiagnosticar este tipo de patología, condicionando la posibilidad de un tratamiento radical y efectivo, tal como sucedió en el caso clínico que describimos.

\section{CASO CLÍNICO}

Paciente de 61 años de edad, alérgico a penicilinas, con antecedentes personales de hipertensión arterial e hiperglucemia, fumador de 30 cigarri- llos/día durante 40 años hasta hace seis años. Cumple criterios clínicos de bronquitis crónica desde hace 10 años.

Encontrándose previamente bien, comenzó hace cuatro meses con un dolor localizado en hombro izquierdo y región interescapular, irradiado a lo largo del brazo por su cara cubital con pérdida de sensibilidad en dedos anular y medio de la mano izquierda. También refería tos escasa sin expectoración. No presentaba fiebre ni anorexia y sólo decía tener una ligera pérdida de peso y sensación de cansancio. Acudió por todo ello a su médico quien le prescribió tratamiento analgésico. Dos meses después, ante la ausencia de mejoría, se le realiza una radiografía de tórax que fue valorada como normal (Fig. 1), no modificándose el tratamiento, recomendándole ejercicios de rehabilitación con un fisioterapeuta al relacionar su sintomatología con una posible patología osteomuscular, encontrándose pendiente de una consulta a un traumatólogo. 

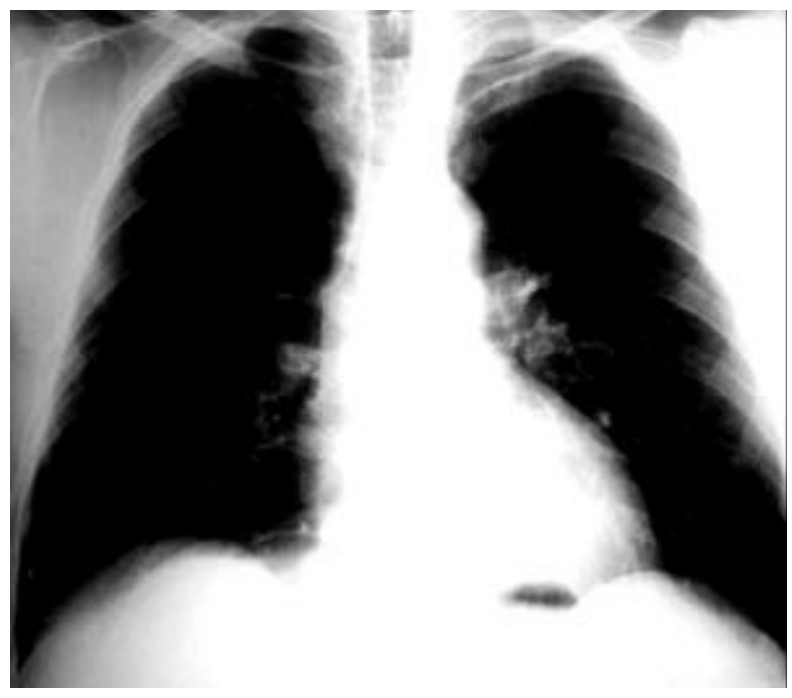

Figura 1

Ante la falta de respuesta clínica se le realiza al paciente una resonancia magnética nuclear con objeto de descartar una hernia cervical, evidenciándose la existencia de una masa en el vértice pulmonar izquierdo (Fig. 2), siendo remitido a nuestro centro para completar el estudio. A la exploración física el paciente presentaba un buen estado general, se evidenció la presencia de miosis en ojo izquierdo con discreta ptosis palpebral y enoftalmos y afección del plexo braquial.

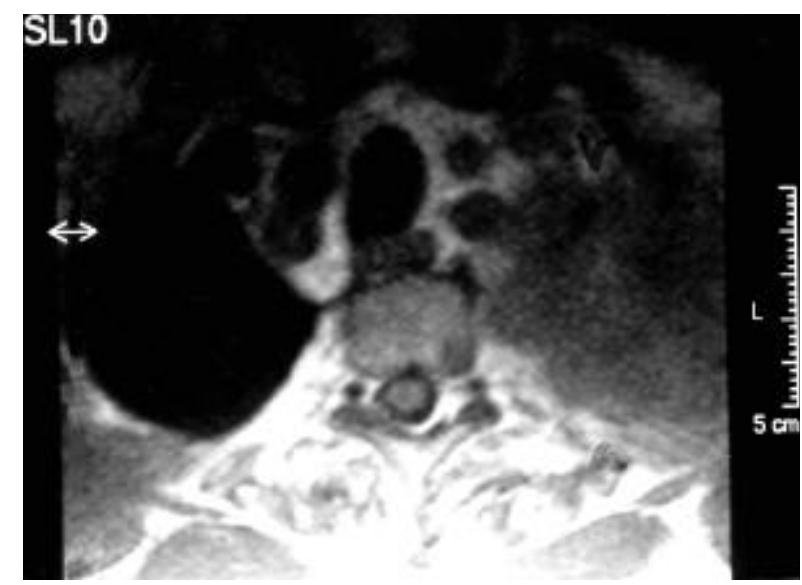

Figura 2

Con respecto a. las exploraciones complementarias realizadas: en la radiografía de tórax se evidenciaba engrosamiento apical izquierdo sin pérdida de volumen, tanto en el estudio que aportaba el enfermo como en la placa realizada al ingreso. La resonancia magnética nuclear cervicotorácica mostraba una masa apical izquierda que se introducía por el agujero de conjunción $\mathrm{D}_{1}$ y $\mathrm{D}_{2}$ englobando la raíz sin compresión medular, observándose una hi- perintensidad completa en $\mathrm{D}_{3}$ y $\mathrm{D}_{4} \mathrm{y}$ parcial en $\mathrm{D}_{1}$, $\mathrm{D}_{2} \mathrm{y} \mathrm{D}_{5}$ por probables metástasis óseas. En la tomografía axial computarizada torácica (TAC) (Fig. 3) se apreciaba una masa con densidad de tejidos blandos en vértice pulmonar izquierdo que invade la pared costal produciendo una lesión lítica en los arcos posteriores de $1^{\mathrm{a}}$ y $2^{\mathrm{a}}$ costillas izquierdas. Además, engrosamiento pleural localizado, discreto derrame pleural izquierdo y lesión lítica en cuerpo vertebral de $\mathrm{D}_{4}$. En la gammagrafía ósea había un aumento de captación en $1^{\mathrm{er}}$ arco costal anterior y $4^{\circ}$ posterior izquierdos atribuidos a posibles metástasis óseas. Broncoscópicamente no se evidenciaron alteraciones en el árbol bronquial, siendo el cepillado bronquial y la citología del broncoaspirado negativas para células tumorales malignas. Se realizó una punción aspiración con aguja fina transtorácica, correspondiendo anatomopatológicamente a un carcinoma pleomórfico, probablemente glandular. El paciente fue diagnosticado de carcinoma de pulmón (tumor de Pancoast) estadio IV $\left(\mathrm{T}_{3} \mathrm{~N}_{0} \mathrm{M}_{1}\right)$, iniciándose tratamiento con radioterapia, sin mejoría alguna y tratamiento paliativo del dolor. La evolución del enfermo fue progresivamente empeorando, con posterior aplastamiento de las vértebras $\mathrm{D}_{3}$ y $\mathrm{D}_{4}$ y compresión medular que condicionó una paresia de extremidades inferiores, arreflexia y pérdida de sensibilidad desde hemiabdomen inferior. Finalmente el paciente falleció seis meses después de la primera consulta.

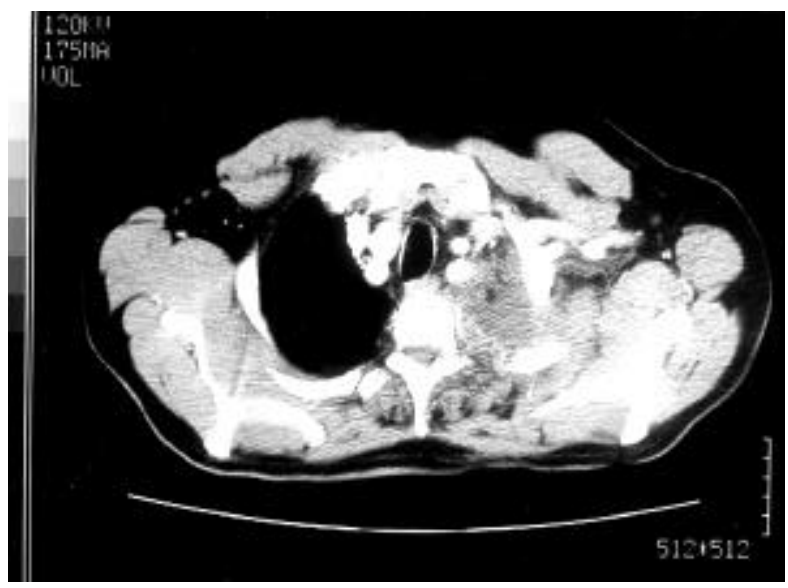

Figura 3

\section{DISCUSIÓN}

El hombro es una estructura compleja y representa la articulación de mayor movilidad de nuestro organismo. Las causas de dolor de hombro son múltiples y pueden tener origen en la propia articulación o fuera de ella, de ahí que sea necesario el conocimiento de la compleja anatomía del hombro (Tabla I). El dolor suele localizarse en el muñón del 
Tabla I

CAUSAS DE DOLOR DE HOMBRO

\begin{tabular}{l} 
PAT OGÍA PERIARTICULAR \\
\hline Tendinitis del manguito/ pinzamiento subacromial \\
Tendinitis cálcica \\
Desgarro del manguito \\
Tendinitis bicipital \\
Artropatía acromioclavicular
\end{tabular}

PATOLOGÍA GLENOHUMERAL

Artritis inflamatoria

Artrosis

Osteonecrosis

Artropatía del manguito

Artritis séptica

Desgarro del labro glenoideo

Capsulitis adhesiva

Inestabilidad glenohumeral

\section{PATOLOGÍA REGIONAL}

Radiculopatía cervical (cervicobraquialgia)

Neuritis braquial

Neuropatías por atraparniento

Artritis esternoclavicular

Distrofia simpática refleja

Patología pleural o hepatobiliar

Infarto de miocardio

Neoplasias: tumores primarios o metastásicos,

Pancoast, mieloma, etc.

hombro y cara externa del brazo y excepcionalmente puede extenderse fuera de esta localización. En general, ante un paciente con dolor de hombro debe hacerse una historia clínica detallada, especialmente teniendo en cuenta las características del dolor, su duración y localización, así como su relación con la actividad diaria. La exploración física es fundamental en el diagnóstico diferencial del hombro doloroso, y debe incluir inspección de zonas atróficas, palpación de las áreas dolorosas, movilidad activa y contra resistencia, comparándolo siempre con el hombro contralateral. También debe examinarse sistemáticamente el cuello y el codo y hacerse una exploración neurológica de los miembros superiores. Si la exploración del hombro es normal debe pensarse en una causa externa al hombro.

Con esta estrategia seguida de una radiografía simple de hombro en los casos de dolor persistente, se consigue el diagnóstico en la mayoría de las ocasiones. La radiografía permite identificar cambios artríticos, calcificaciones tendinosas, osteonecrosis y un gran porcentaje de neoplasias. Sólo una pequeña parte de los pacientes requerirán otras técnicas complementarias como ecografía, resonancia magnética o tomografía axial computarizada (TAC).

En aquellos casos en los que los movimientos de la articulación activos, pasivos o resistidos no re- produzcan el dolor y sobre todo cuando existan signos de compromiso radicular o de plexo, deben investigarse las causas de dolor referido, en particular, patología cervical o intratorácica. En ambas circunstancias, también la radiología simple suele ser la primera prueba complementaria a solicitar. El tumor de Pancoast es la enfermedad neoplásica más común no metastásica que compromete el plexo braquial, de ahí que deba tenerse en consideración en el diagnóstico diferencial del hombro doloroso. Se trata de una forma de presentación muy conocida del carcinoma de pulmón, sin embargo es frecuentemente infradiagnosticado, como consecuencia de sus características clínicas y radiológicas.

El tumor de Pancoast fue descrito originalmente en $1924^{2}$ y su frecuencia oscila entre el 3-5\% de todos los tumores pulmonares. Es un tumor habitualmente bien localizado, que se sitúa a nivel del vértice pulmonar, por encima de la impronta que deja en el pulmón la arteria subclavia (tumor del sulcus). Característicamente va a dar origen a una constelación de signos y síntomas, debido a la invasión y/o compresión de las estructuras vecinas: arteria y vena subclavias, la arteria carótida común, los nervios vago, frénico y recurrente laríngeo, cadena simpática y ganglio estrellado, cuerpos vertebrales y costillas. La etiología del síndrome de Pancoast es neoplásica en la mayoría de las ocasiones, constituyendo el carcinoma broncogénico la causa más habitual, siendo los tipos histológicos más comunes el epidermoide y el adenocarcinoma.

El tumor de Pancoast clínicamente puede dar lugar a un dolor localizado en el hombro, de características radiculares, irradiado a lo largo del brazo y parestesias, como consecuencia de la infiltración del plexo braquial. En la exploración física destaca la presencia de un síndrome de Horner (ptosis palpebral, enoftalmos y miosis), debilidad y atrofia de los músculos intrínsecos de la mano.

Es de destacar que radiológicamente se manifiesta como una opacidad o engrosamiento unilateral localizada en el vértice pulmonar, que puede ser fácilmente no reconocido debido a que en ocasiones puede mostrarse como un mínimo engrosamiento pleural apical o bien atribuirse a lesiones apicales inespecíficas. Ante la duda, puede ser útil la proyección lordótica. El TAC torácico y la resonancia magnética nuclear permiten valorar mejor su extensión. El tratamiento habitual es la radioterapia preoperatoria seguida de cirugía. La supervivencia a los cinco años alcanza el $35 \%$.

Es importante señalar que la radiografía de tórax es el procedimiento fundamental en el diagnóstico de sospecha del carcinoma de pulmón, ya que tiene una eficacia global muy elevada. Lamentablemente, en ocasiones las alteraciones radiológicas pueden pasar fácilmente desapercibidas para el clínico. Es el caso del tumor de Pancoast, que como conse- 
cuencia de su sintomatología extrapulmonar hace que la radiografía de tórax no sea adecuadamente examinada, lo que motiva que estos pacientes sean derivados inicialmente a la consulta de traumatología o sean tratados erróneamente durante largo tiempo de patología osteomuscular (bursitis, osteoartritis cervical, hernia discal), implicando en un gran número de ocasiones un retraso en el diagnós tico, que puede llegar a alcanzar hasta los cinco o diez meses ${ }^{4 \cdot 6}$, lo cual va a tener importantes implicaciones en el tratamiento y pronóstico de estos pacientes. Así, Bisbinas y cols. ${ }^{6}$, describen recientemente cuatro casos de pacientes con sintomatología localizada en el hombro, cuello, braquialgia o sintomatología neurológica que fueron erróneamente derivados al departamento de Cirugía Ortopédica, siendo diagnosticados posteriormente de un tumor de Pancoast con un retraso medio de 18,5 meses desde el inicio de los síntomas. Como principal causa de este retraso en la derivación del paciente se señala la ausencia de sospecha diagnóstica por parte del médico, por lo que los pacientes son tratados con un presunto diagnóstico de bursitis de hombro, espondilosis cervical o síndrome del orificio torácico superior, y algunos incluso fueron intervenidos innecesariamente. Esto hace insistir en la necesidad ante un hombro doloroso persistente en realizar una exploración neurológica adecuada y valorar la existencia de lesiones pulmonares en la radiografía de tórax.

Pero esta dificultad diagnóstica y el retraso asociado no es exclusivo del tumor de Pancoast, sino que se puede atribuirse globalmente al cáncer de pulmón ${ }^{7}$. Se ha recomendado que el intervalo de tiempo entre la primera consulta del paciente con su médico y la resección pulmonar, no debe ser mayor de 6-8 semanas. Sin embargo, en la práctica habitual el tiempo transcurrido desde el inicio de los síntomas hasta su remisión a un especialista puede ser indeseablemente largo, llegando a alcanzar hasta tres o cuatro meses el tiempo que pasa desde el inicio del cuadro hasta cuando es visto por un cirujano torácico ${ }^{8}$. Milleron ${ }^{9}$ encuentra en Francia, en un estudio prospectivo en 72 pacientes, un retraso medio importante desde la presentación de los síntomas hasta la primera consulta (103 días) y de la presentación hasta el tratamiento de 155 días, datos muy similares a los obtenidos en otros países. En nuestro medio ${ }^{10}$ la demora media entre la sospecha diagnóstica y la toracotomía fue de 45 días, siendo en ocasiones superior a 90 días, lo que ha sido considerado como tiempos elevados y por tanto susceptibles de mejoría.

Por todo ello, con el objetivo de mejorar el diagnóstico del carcinoma de pulmón y reducir en lo posible retrasos muchas veces inaceptables, es necesario mantener un alto índice de sospecha por parte del médico de asistencia primaria, especialmente en pacientes de riesgo, así como establecer programas de educación sanitaria entre los pacientes fumadores.

\section{CORRESPONDENCIA:}

F. del Campo Matías

Padre Francisco Suárez, 8-5º dcha.

47006 Valladolid

\section{Bibliografía}

1. Woodward TW, Best TM. The painful shoulder: Clinical evaluation. Am Fam Physician 2000; 61: 3079-88.

2. Pancoast HK. Importance of careful roetgen-ray investigation of apical chest tumors. JAMA 1924 83:1407-14.

3. Arcasoy SM, Jett JR. Superior pulmonary sulcus tumors and Pancoast's syndrome. N Engl J Med 1997; 33: 1370-6

4. Delaney TF, Nielsen GP. Case Records of the Massachusetts General Hospital (Case 18-2000): A 45year-old woman with a thoracic mass and Pancoast's syndrome N. Engl J Med 2000; 342 (24): 1814-21.

5. Bonaventura I, Mir I, Marcos JA, Bastus R, Rami R, Martinez I, et al. Tumor de Pancoast: dificultades para su diagnóstico. Rev Clin Esp 1993; 435-7.

6. Bisbinas I, Langkamer VG. Pitfalls and delay in the diagnosis of Pancoast tumor presenting in orthopaedic units. Ann R Coll Surg Engl 1999; 81: 291-5.

7. George PJM. Delays in the management of lung cancer. Thorax 1997; 52: 107-8.

8. Wells FCB. Delays in the diagnosis and surgical treatment of lung cancer. Thorax 1996; 51: 903-6.

9. Milleron B, Mangiapan G, Terrioux PH, Guigay J, Mayaud $C$. Delay in the diagnosis and treatment of lung cancer. Thorax 1997; 52: 398.

10. Encuentra A, Martín de Nicolas JL, Casado López, de Miguel Poch E, Marrón Fernández C y Grupo Cooperativo de Carcinoma Broncogénico de SEAPAR. 